\title{
Comparison of several tetrahedra-based lattices
}

\author{
Maged Elhajal, Benjamin Canals, Claudine Lacroix \\ Laboratoire Louis Néel, 25 avenue des Martyrs, BP 166, 38042 Grenoble Cedex 9, \\ France \\ June 2000
}

\begin{abstract}
A comparison of the quantum Heisenberg antiferromagnetic model (QHAM) on the pyrochlore lattice, the checkerboard lattice and the square lattice with crossing interactions is performed. The three lattices are constructed with the same tetrahedral unit cell and this property is used to describe the low energy spectrum by means of an effective hamiltonian restricted to the singlet sector. We analyze the structure of the effective hamiltonian and solve it within mean field approximation for the three lattices.
\end{abstract}




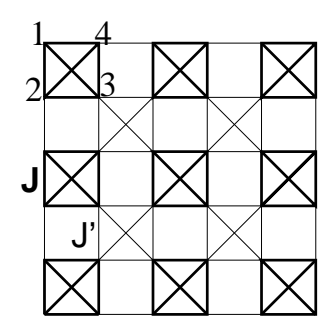

(a)

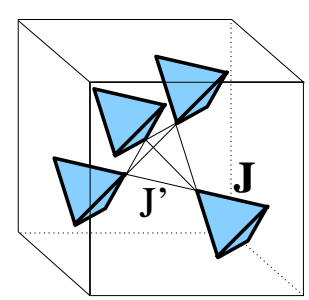

(b)

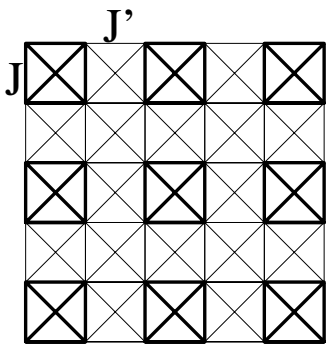

(c)

Figure 1: Checkerboard (a), pyrochlore (b) and square lattice with crossing interactions. Bold lines represent intra-TT $(J)$ interactions treated exactly, and thin lines represent inter-TT $\left(J^{\prime}\right)$ interactions considered as a perturbation.

\section{Introduction}

Geometrical frustration gives rise to original magnetic properties at low temperature: on some lattices, a continuous degeneracy of the classical ground state is obtained in the mean field approximation, preventing any phase transition to a long range ordered state 1 . We will focus here on the quantum spin- $\frac{1}{2}$ Heisenberg model on the checkerboard, the pyrochlore and the square lattice with crossing interactions and specially on their low energy spectrum.

The checkerboard lattice is a square lattice of tetrahedra (TT) (see figure 17a), while the pyrochlore lattice is a fcc lattice of TT (see figure 1 $1 \mathrm{~b}$ ). The checkerboard can be considered as the $2 \mathrm{D}$ analog of the pyrochlore since both of them are made of corner sharing tetrahedra. The square lattice with crossing interactions is also a $2 \mathrm{D}$ lattice but the $\mathrm{TT}$ are more connected than in the checkerboard (see figure 1c).

There is strong evidence, both experimentally 2] and theoretically [3 that the ground state for the QHAM on the pyrochlore lattice is a quantum spin liquid, with a correlation length which hardly exceeds a few interatomic distances. Moreover, it is believed that the low lying excitations are singlet-singlet as in the kagome lattice $\llbracket$. Given these properties, we develop an effective model restricted to the singlet sector of the Hilbert space and taking into account the short range correlations of the spin liquid state. For the checkerboard, it was shown[5] that the ground state is a singlet state. The square lattice with cross- 
ing interactions is similar to the $J_{1}-J_{2}$ model with $J_{1}=J_{2}$. The ground state for this model is not clearly identified[6] for $0.4 \lesssim \frac{J_{2}}{J_{1}} \lesssim 0.6$ but in our case $\left(J_{1}=J_{2}\right)$, the ground state is a columnar dimer state[7].

\section{Effective hamiltonians}

The ground state of one TT is twofold degenerate (2 singlet states). We restrict the accessible states for each TT to these two singlets, and associate a pseudospin $\vec{T}$ (with $\mathrm{T}=\frac{1}{2}$ ) to each $\mathrm{TT}$, the two states $\left(T^{z}= \pm \frac{1}{2}\right)$ of the pseudo-spin represention being the two singlet states. Doing this, we have only taken into account (exactly) the interactions (J) within one TT. We label the 4 sites of each TT as shown in figure 17a. We can choose the two states of the $\mathrm{S}=0$ subspace so that

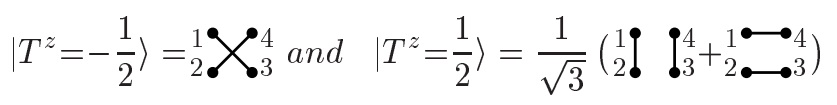

where $\mathrm{a} \bullet \mathrm{b}$ represents a 2 spins singlet $\frac{1}{\sqrt{2}}(|a \uparrow b \downarrow\rangle-|a \downarrow b \uparrow\rangle) .\left|T^{z}=-\frac{1}{2}\right\rangle$ describes a dimerized state whereas $\left|T^{z}=\frac{1}{2}\right\rangle$ is a linear combination of dimerized states, which is a quadrimer. We then consider the interactions between the tetrahedra $\left(J^{\prime}\right)$ as a perturbation. Since the two singlets of one TT are degenerate, perturbation theory leads to a matrix which we write down in terms of pseudo-spins operators $\left(T^{x}, T^{y}\right.$ and $\left.T^{z}\right)$. This leads to an effective hamiltonian in pseudo-spins space. These hamiltonians have been calculated up to third order in $\lambda=\frac{J^{\prime}}{J}$ for all lattices. For the three lattices at first order, there is no effective interaction (the effective hamiltonian is a spherical matrix) because the operators in real spin space $\mathbf{s}_{\mathbf{i}} \mathbf{s}_{\mathbf{j}}$ have non-zero matrix elements only between singlet and triplet states.

For the checkerboard lattice, we find (with $J^{\prime}=J$ and summing the contributions up to third order):

$$
\begin{aligned}
\frac{H}{|J|} & =\sum_{\text {psd-spins }}-\frac{85}{192}-\frac{1}{24} T_{i}^{z} \\
& +\sum_{\text {horiz }} \frac{1}{16} T_{i}^{z} T_{j}^{z}-\frac{1}{16} T_{i}^{x} T_{j}^{x}-\frac{1}{16 \sqrt{3}}\left(T_{i}^{z} T_{j}^{x}+T_{i}^{x} T_{j}^{z}\right) \\
& +\sum_{\text {verti } i} \frac{1}{16} T_{i}^{z} T_{j}^{z}-\frac{1}{16} T_{i}^{x} T_{j}^{x}+\frac{1}{16 \sqrt{3}}\left(T_{i}^{z} T_{j}^{x}+T_{i}^{x} T_{j}^{z}\right) \\
& +\sum_{N N N}-\frac{1}{48} T_{i}^{z} T_{j}^{z}+\frac{1}{16} T_{i}^{x} T_{j}^{x} \\
& +\sum_{\text {triangles }} \frac{1}{24} T_{i}^{z} T_{j}^{z} T_{k}^{z}+\frac{1}{8 \sqrt{3}}\left(T_{i}^{z} T_{j}^{z} T_{k}^{x}-T_{i}^{z} T_{j}^{x} T_{k}^{z}\right) \\
& -\frac{1}{8} T_{i}^{z} T_{j}^{x} T_{k}^{x}
\end{aligned}
$$




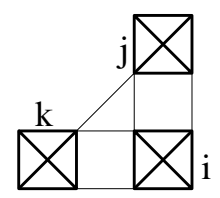

Figure 2: Three pseudo-spins interactions in the checkerboard include all possible triangles like this one and those obtained by reflexion with respect to the $x$ and $y$ axes, keeping always the same labelling $(i, j, k)$ for the pseudo-spins (see fifth sum of the effective hamiltonian)

where the first sum is over pseudo-spins, which are on a square lattice for the checkerboard. The second and third sums are over horizontal (x axis) and vertical (y axis) nearest neighbour interactions, whereas the fourth sum is over second nearest neighbours. The fifth sum is over all possible triangles and the pseudo-spins are then labelled as shown in figure 2.

The 'field' acting on pseudo-spins has nothing to do with a real magnetic field and it is hopeless to measure any 'real' magnetization, since all states in our representation are non magnetic. The interactions along $x$ and $y$ directions of the square lattice are slightly different. This broken symmetry has been introduced artificially when we have choosen two arbitrary singlets for each TT which are not invariant under rotation of $\frac{\pi}{2}$, but the final result does not depend on this choice. The second and third sums lead to anisotropic exchange and to cross terms. There are three pseudo-spins interactions at third order in $\lambda$. The higher the order of perturbation is, the higher the number of spins involved in the interactions will be. However, since the correlation length is short, it is believed that taking into account the lowest orders in perturbation will be enough to catch the essential feature of the spin liquid behaviour.

The 'field' in pseudo-spins space can be interpreted as follows: since the state $\left|T^{z}=-\frac{1}{2}\right\rangle$ corresponds to a dimerized singlet and the state $\left|T^{z}=\frac{1}{2}\right\rangle$ to a quadrimer (see (1)), a negative 'field' would be a hint that the system has a tendency to dimerize, whereas a positive 'field' would be a tendency of the system to be in a 4-spin-singlet (quadrimerization). This is confirmed by the expression of the effective hamiltonian for the square lattice with crossing interactions (at third order in $\lambda$ ) which is known to dimerize (we have set $J^{\prime}=$ $J)$ :

$$
\begin{aligned}
\frac{H}{|J|}= & \sum_{\text {psd-spins }}-\frac{125}{192}+\frac{5}{6} T_{i}^{z} \\
& +\sum_{\text {horiz }}-\frac{5}{24} T_{i}^{z} T_{j}^{z}-\frac{5}{8} T_{i}^{x} T_{j}^{x}-\frac{5}{8 \sqrt{3}}\left(T_{i}^{z} T_{j}^{x}+T_{i}^{x} T_{j}^{z}\right) \\
& +\sum_{\text {verti }}-\frac{5}{24} T_{i}^{z} T_{j}^{z}-\frac{5}{8} T_{i}^{x} T_{j}^{x}+\frac{5}{8 \sqrt{3}}\left(T_{i}^{z} T_{j}^{x}+T_{i}^{x} T_{j}^{z}\right) .
\end{aligned}
$$


This hamiltonian was calculated at second order by Kotov, Zhitomirsky and Sushkov[7]. It is found that the hamiltonian at third order is equivalent to the hamiltonian at second order multiplied by a constant $\left(\frac{1}{4}\right)$, so including the third order will not change the structure of the hamiltonian. It is similar to the hamiltonian for the checkerboard at second order except that the field is higher and of opposite sign. In particular, the ratio of the field term on the interactions terms remains constant when including third order, which reflects the tendency of this system to dimerize (because the 'field' is strong enough and negative, so that the pseudo-spins will rather be in the state $\left|T^{z}=-\frac{1}{2}\right\rangle$ which is a dimer). On the contrary, in the checkerboard case, inclusion of third order perturbation decreases the ratio between 'field' and exchange. Whether this is still true at fourth order is being tested. The sign of the field in the checkerboard is a hint of quadrimerization

The hamiltonian for the pyrochlore has already been published in 8. Our expression of the effective hamiltonian is slightly different (the constant term is different). The main difference with the effective hamiltonian for the checkerboard is the absence of 'field' up to third order in $\frac{J^{\prime}}{J}$, and the absence of interactions at second order. The three pseudo-spins interactions are the same as for the checkerboard. Labelling three pseudo-spins interactions is more complex than for the checkerboard case because the pseudo-spins are on a 3D (fcc) lattice.

\section{Mean field approximation}

We have solved the effective hamiltonians for the three lattices within mean field approximation. We have first looked for single-q solutions, taking into account only two pseudo-spins interactions. For the pyrochlore and the checkerboard lattices, it is found that the structure of the ground state is antiferromagnetic in pseudo-spin space.

For the checkerboard lattice, the propagation wave vector is $(\pi, \pi)$. The two sublattices have a pseudo-magnetization which is along $\pm z$. Going back to real spin space, this means that on one sublattice the TT are in a dimerized state (corresponding to pseudo-spin $\left|T^{z}=-\frac{1}{2}\right\rangle$ ), whereas the TT on the other sublattice are quadrimers (in pseudo-spin space: $\left|T^{z}=\frac{1}{2}\right\rangle$ ).

For the square lattice with crossing interactions, all the TT are in the $\mid T^{z}=$ $\left.-\frac{1}{2}\right\rangle$ state at zero temperature, which correspond to a fully dimerized state, as expected[7].

For the pyrochlore lattice, the wave vector can be either $(2 \pi, 0,0),(0,2 \pi, 0)$ or $(0,0,2 \pi)$. The structure is still antiferromagnetic (one dimerized and one quadrimerized sublattices), but the direction of the pseudo-magnetization depends on the wave vector. Each wave vector corresponds to one of the three possible dimerized states for a TT: ${ }_{2}^{1} \chi_{3}^{4},{ }_{2}^{1} \prod_{3}^{4}$ and ${ }_{2 \bullet 3}^{1 \bullet}{ }_{2}$. In these antiferromagnetic single-q configurations, the contribution of the three pseudo-spins interactions vanishes for both lattices.

For the pyrochlore lattice we have then looked for multi-q structures as done 
in Ref. [8]. A four sublattices stucture is found to be favoured by three pseudospins interactions. It corresponds to a triple-q structure which is a superposition of the three single-q solutions. Three of the sublattices are in the three possible dimerized states of a TT, and the pseudo-magnetization of the fourth one is: $\left\langle T_{i}^{x}\right\rangle=\left\langle T_{i}^{z}\right\rangle=0$ and $\left\langle T_{i}^{y}\right\rangle= \pm \frac{1}{2}$. The sign of $\left\langle T_{i}^{y}\right\rangle$ on each site is not fixed since the energy is independent of $T^{y}$ and this sublattice is disordered.

Separating the contributions of the different orders of perturbation, the total energy per TT (or pseudo-spin) for this triple-q solution is:

$$
E=-\frac{3}{2}|J|-\frac{9}{16} \frac{J^{\prime 2}}{|J|}+\frac{9}{128} \frac{\left|J^{\prime 3}\right|}{J^{2}}
$$

The modulus of the different energy contributions decrease as the order of perturbation increases, as expected. Fixing $\frac{J^{\prime}}{J}=1$, this gives an energy per site $\left(\frac{E}{4}=-\frac{255}{512}|J| \simeq-0.49805|J|\right)$ slightly lower than the value obtained in Ref. [8] $\left(\frac{E}{4} \simeq-0.48763|J|\right)$. The energy per site obtained for the checkerboard lattice is $\frac{E}{4} \simeq-0.49622|J|$

To summarize, our results indicate that the effective hamiltonians are different for the pyrochlore, the checkerboard and the square lattice with crossing interactions, due to the different geometries. The 'field' in the effective hamiltonian is associated to dimerization or quadrimerization. The energies obtained for the ground states in the pyrochlore lattice (3D) and the checkerboard lattice (2D) are quite similar. However, the singlet structures of the two lattices are different: the pyrochlore lattice has a four sublattices structure, one of which does not order, the three other being in dimerized states. The square lattice with crossing interactions has a clearly different behaviour at low temperature.

Further studies are necessary to see whether the structures obtained persist if fluctuations around mean field solution and higher order terms are taken into account.

\section{acknowledgments}

It is a pleasure to acknowledge valuable discussions with Pr. A.B. Harris and Pr. A.J. Berlinsky.

\section{References}

[1] J.N. Reimers, A.J. Berlinsky and A.-C. Shi, Phys. Rev. B 43, 865 (1991)

[2] R. Ballou, E. Lelièvre-Berna and B. Fåk, Phys. Rev. Lett. 76, 2125 (1996).

[3] B. Canals and C. Lacroix, Phys. Rev. Lett. 80, 2933 (1998).

[4] P. Lecheminant, B. Bernu, C. Lhuillier, L. Pierre and P. Sindzingre, Phys. Rev. B 56, 2521 (1997)

[5] E.H. Lieb and P. Schupp, Phys. Rev. Lett. 83, 5362 (1999) 
[6] R.R.P. Singh, Z. Weihong, C.J. Hamer and J. Oitmaa, Phys. Rev. B 60, 7278 (1999)

[7] V.N. Kotov, M.E. Zhitomirsky and O.P. Sushkov, Cond. Mat./0001282

[8] A.B. Harris, A.J. Berlinsky and C. Bruder, J. Appl. Phys. 69, 5200 (1991) 